\title{
SEARCH \& INSPECTION ARCHAEOLOGICAL UNDERWATER CAMPAIGNS IN THE FRAMEWORK OF THE EUROPEAN ARROWS PROJECT
}

\author{
B. Allotta ${ }^{1,5}$, R. Costanzi ${ }^{3,5}$, F. Mugnai ${ }^{7,8}$, M.Reggiannini $^{4}$, A. Ridolfi ${ }^{1,5}$, D. Scaradozzi ${ }^{2,5,6}$ \\ ${ }^{1}$ Department of Industrial Engineering, University of Florence, Florence, Italy benedetto.allotta@unifi.it, a.ridolfi@ unifi.it \\ ${ }^{2}$ Dip. di Ingegneria dell'Informazione, Università Politecnica delle Marche, Ancona, Italy \\ ${ }^{3}$ Dipartimento di Ingegneria dell'Informazione e Centro di ricerca "E. Piaggio", Università di Pisa, Pisa, Italy, \\ riccardo.costanzi@unipi.it \\ ${ }^{4}$ Institute of Information Science and Technologies - CNR Pisa, Italy \\ ${ }^{5}$ Interuniversity Center of Integrated Systems for the Marine Environment (ISME), Italy, http://www.isme.unige.it \\ ${ }^{6}$ Laboratoire des Sciences de l'Information et des Systemes - Marseille, France \\ ${ }^{7}$ Department of Civil and Environmental Engineering, University of Florence, Florence, Italy \\ ${ }^{8}$ European Commission, Joint Research Centre (JRC), Directorate A - Scientific Development Unit, \\ francesco.mugnai@ec.europa.eu
}

\section{Commission II, WG II/8}

KEY WORDS: Autonomous Underwater Vehicles, Underwater archaeology, Underwater 3D reconstruction, Marine robotics, Underwater surveys

\begin{abstract}
:
Autonomous Underwater Vehicles (AUVs), benefiting from significant investments in the past years, are commonly used for military security and offshore Oil\&Gas applications. The ARROWS project ${ }^{1}$, aimed at exporting the AUV technology to the field of underwater archaeology, a low-budget research field compared to the previous ones. The paper focuses on the strategy for vehicle coordination adopted within the project, a Search and Inspection (S\&I) approach borrowed from the defense field (e.g., mine countermeasure - MCM) that proved to be an efficient solution also for the main phases of an underwater archaeological mission. The other main novelty aspect is represented by MARTA (MArine Robotic Tool for Archaeology) AUV: it is a modular vehicle easily and quickly reconfigurable developed in the framework of ARROWS according to the project Archaeological Advisory Group (AAG) guidelines. Results from the final demonstration of the project, held in Estonia during Summer 2015, are proposed in the paper as an experimental proof of the validity of the proposed S\&I strategy, and MARTA functioning and its adaptability to the mission requirements. Even in its first prototype version, MARTA successfully played the Inspection role within the AUV team, collaborating with a commercial Search AUV. Acoustic and optical data collected during the mission and processed to increase their intelligibility for the human operator are proposed and discussed.
\end{abstract}

\section{INTRODUCTION}

The methodology that led the researchers during the three years of ARROWS was based on the guidelines issued by the project Archaeology Advisory Group (AAG), composed of many European archaeologists: some of them belonged to the ARROWS consortium, some others were invited to join the AAG. The ARROWS project proposed to adapt and develop low-cost Autonomous Underwater Vehicle (AUV) technologies to significantly reduce the expense of archaeological operations, covering the full extent of an underwater campaign (Allotta et al., 2015a). High-budget research field applications like military security and offshore Oil\&Gas can already benefit from the use of reliable AUV technology for different tasks with advantages in terms e.g. of necessary time, mission costs and human safety (AUVAC Database, 2016), (OceanServer IVER3, 2016). This was possible thank to significant investments made in the recent past. The underwater archaeology field (as well as other low budget research fields, e.g. biology or geology) cannot easily exploit the same technology because of prohibitive costs. ARROWS goal was to answer the requests of the underwater archaeologists providing cost affordable solutions, using AUVs.
Current approaches adopted by underwater archaeologists mainly consist of offset measurements, tape measure trilateration and simple photography (Van Damme, 2015). Under particular circumstances, especially when cultural heritages insist on water and land at the same time, like bridges and docks, a multi-disciplinary approach is commonly needed. Having an autonomous underwater facility to be used as integration of the most common techniques e.g. Laser Scanning, Geotechnical investigation, underwater inspections etc. (Mugnai et al., 2019), could be considered as a strong add value. In addition to the intrinsic safety matters related to the direct involvement of human operators in the data capture process in a potentially dangerous environment, some of the tasks performed by archaeologists typically suffer from the time consuming issues and relevant risk of human errors within the hostile working scenario. Currently, many archaeological campaigns, mainly, make use of divers (Skarlatos et al., 2012), (Drap, 2012), (Henderson et al., 2011) and, sometime, of ROVs (Remotely Operated Vehicles). In this context, ROVs and divers have been exploited many times, e.g., during the European project VENUS (Conte et al., 2005). Dedicated methodologies, small ROVs, and tools have been developed to help archaeologists during their work (Sorbi et al., 2015).

\footnotetext{
${ }^{1}$ http://www.arrowsproject.eu/
} 
AUVs represent a natural evolution of ROVs. Using reliable AUV can actively help in reducing the costs of the logistics (e.g., dedicated personnel piloting the vehicle is not needed, differently with respect to ROVs). In order to fulfil the AAG declared needs, the ARROWS technological researchers developed, among several other tools, MARTA (MArine Robotic Tool for Archaeology) AUV (Allotta et al., 2015b).

The other adopted solution is the use of a cooperating team of heterogeneous AUVs with the double benefit of simpler and, thus, cheaper vehicles and of time-saving thanks to the possibility of tasks parallelization. When more vehicles are used during the same mission a clear strategy for task division and coordination is needed. In the future, teams of AUVs could communicate and share common strategies, or react on a common goal, with the typical MultiAgents Systems strategy, adopted for not supervised control situations (Morganti et al., 2009). ARROWS missions were conducted with a Search-andInspect (S\&I) approach, involving two different specialized vehicles (the commercial IVER3 by OceanServer as SearchAUV and MARTA as Inspection-AUV). The Search-andInspect (S\&I) approach is similar to the search-classify-map, and reacquire-identify strategy developed and used by military Mine CounterMeasures (MCM) operations as described in (Freitag et al., 2005).

Some of the goals achieved in the ARROWS project thanks to the use of autonomous technologies include: fast and low cost horizontal surveys of large areas using customised AUVs with multimodal sensing; high quality maps from better image reconstruction methods and better localization abilities of AUVs; "Shipwreck penetration" and internal mapping using small low cost vehicles localizing using fixed pingers; monitoring of changes via back-to-the-site missions. Furthermore, an effort in providing the end-users of the ARROWS system (underwater archaeologist teams) with a tool (Graphical User Interface - GUI) for mission planning and monitoring that does not require the constant presence of technological professionals was one of the requirements of the AAG.

\section{EXPERIMENT DESCRIPTION AND ACHIEVED RESULTS}

The ARROWS project demonstration was composed of different on-field campaigns. It is aimed at the verification of the developed technologies effectiveness in the different sea environments characterizing Europe. In this paper, the inspection campaign on the Baltic Sea is reported.

The scenarios were represented by some well-known archaeological sites in the Bay in front of Tallinn and by the Rummu quarry (Figure 1), a mining site for Vasalemma marble (a kind of limestone). The quarry, which has been affected by catastrophic flooding, is still completely submerged. The quarry develops mainly along the West-East direction for about 2, 5 $\mathrm{km}$. It is frequented by a lot of swimmers and divers that use to explore the Western part of the lake because of its greater attractiveness due to some partly submerged trees and buildings that can be easily explored inside.

The results presented in this paper are related to missions conducted between July $20^{\text {th }}$ and July $22^{\text {nd }} 2015$. The above mentioned Search and Inspection strategy was here applied and validated for an archaeology oriented mission based on the collaboration of a heterogeneous team of AUVs composed of a Side Scan Sonar (SSS) equipped commercial AUV playing the Searching role and MARTA AUV (Figure 2), equipped with a High Definition camera playing the Inspection role.

\subsection{Search and Inspection}

From the analysis of the SSS sonograms acquired by the searching AUV, two unidentified targets were localized. The GPS coordinates of the two targets were retrieved through the analysis of the collected acoustic images.

Inspection campaign was planned with MARTA AUV following the acquired acoustic targets' coordinates that have been previously delivered by the searching AUV. Inspection campaign was aimed at collecting optical images through its High Definition camera on the two spots. The reference path and all the necessary mission parameters were defined through the graphical HMI developed within the project to simplify the interaction of a non-specialized end-user with the developed tools2. The mission was composed of two different lawnmower submissions: the first one with path legs orthogonal to the line connecting the two target points and the second one with path legs parallel to it. The layout of the two submissions is represented in Figure 3.

The characteristics of the planned path have been decided according to mission exigencies in terms of reference altitude (was set at $3.0 \mathrm{~m}$ ), the line-space (was set at $1.5 \mathrm{~m}$ for the first mission and $1.0 \mathrm{~m}$ for the second one). The reference speed for inspecting AUV was set at $2.0 \mathrm{kn}(\approx 1 \mathrm{~m} / \mathrm{s})$.

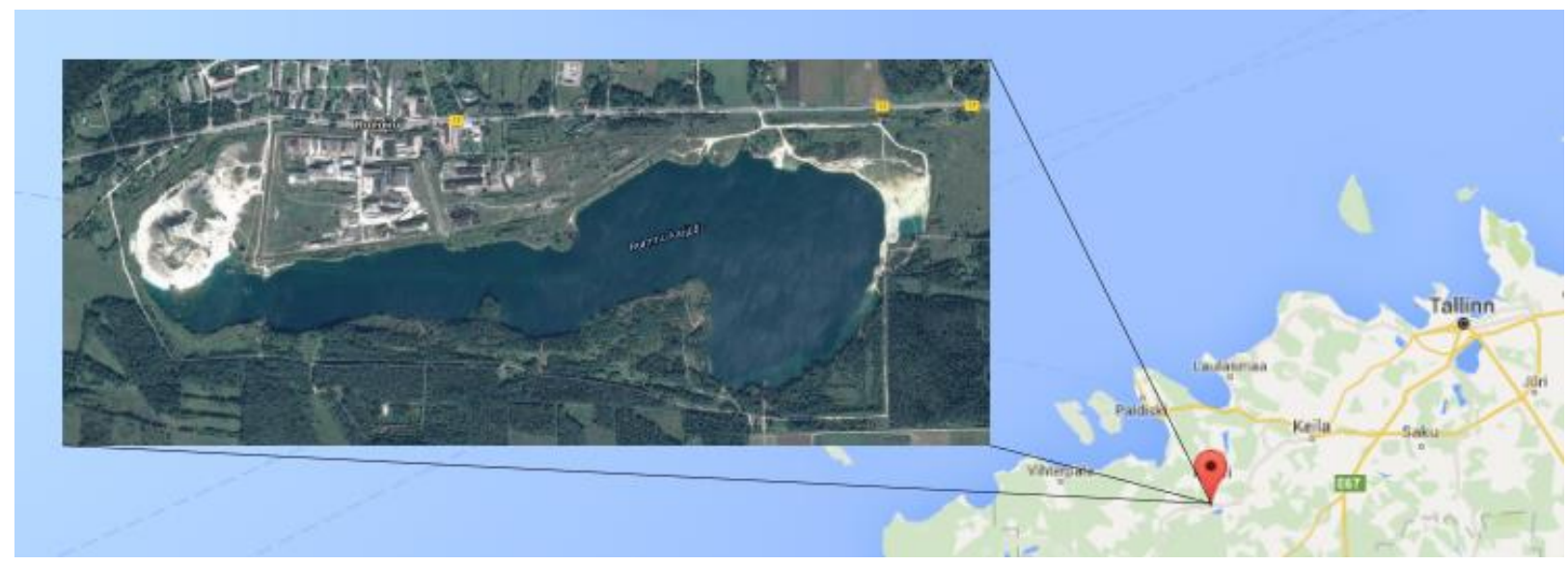

Figure 1. Rummu quarry with respect to Tallinn on Google Maps - Map data @2016 Google

${ }^{2}$ A video tutorial describing the usage simplicity of the ARROWS HMI was produced in the framework of the project and is available at the link: https: //www youtube. com/watch? $v=$ fHNRCCMtgFE 


\subsection{Optical data processed for 3D reconstruction}

Following the approach outlined in (Allotta et al., 2015c), (Allotta et al., 2015d), (Conte et al., 2012), the first instances of the Rummu quarry survey operations had the primary goal of detecting those regions of the seabed that featured substantial clues for the presence of interesting objects. By using AUVs, acoustic positioning systems and improved photo-cameras, it has been possible to obtain, in addition to $2 \mathrm{D}$ mosaics of the inspected area (Martin et al., 2002), (Pizarro et al., 2003), (Ludvigsen et al., 2007), a very precise geo-referenced map or 3D geo-referenced reconstruction of large areas of the seabed. Maps obtained through correlation of optical, acoustic, and positioning data are an innovative and essential tool because

$$
\mathbf{X}_{\mathrm{ij}} \propto \mathbf{P}_{\mathrm{j}} \mathbf{X}_{\mathrm{i}}
$$

where $\mathrm{Pj}$ is the camera matrix and represents the knowledge about the camera intrinsic parameters (aspect ratio, skew, focal length and camera center coordinates) and extrinsic parameters (the rotation and translation were defining camera pose and position w.r.t. the global world reference frame). A crucial step to pursue the mentioned goal is the detection of those image points xij that are generated by the projection of the same $3 \mathrm{D}$ world point onto different camera planes. This has been obtained by adopting robust methods for the detection and matching of stable features, such as those based on the SIFT features detector (Lowe, 2004). The Structure from Motion

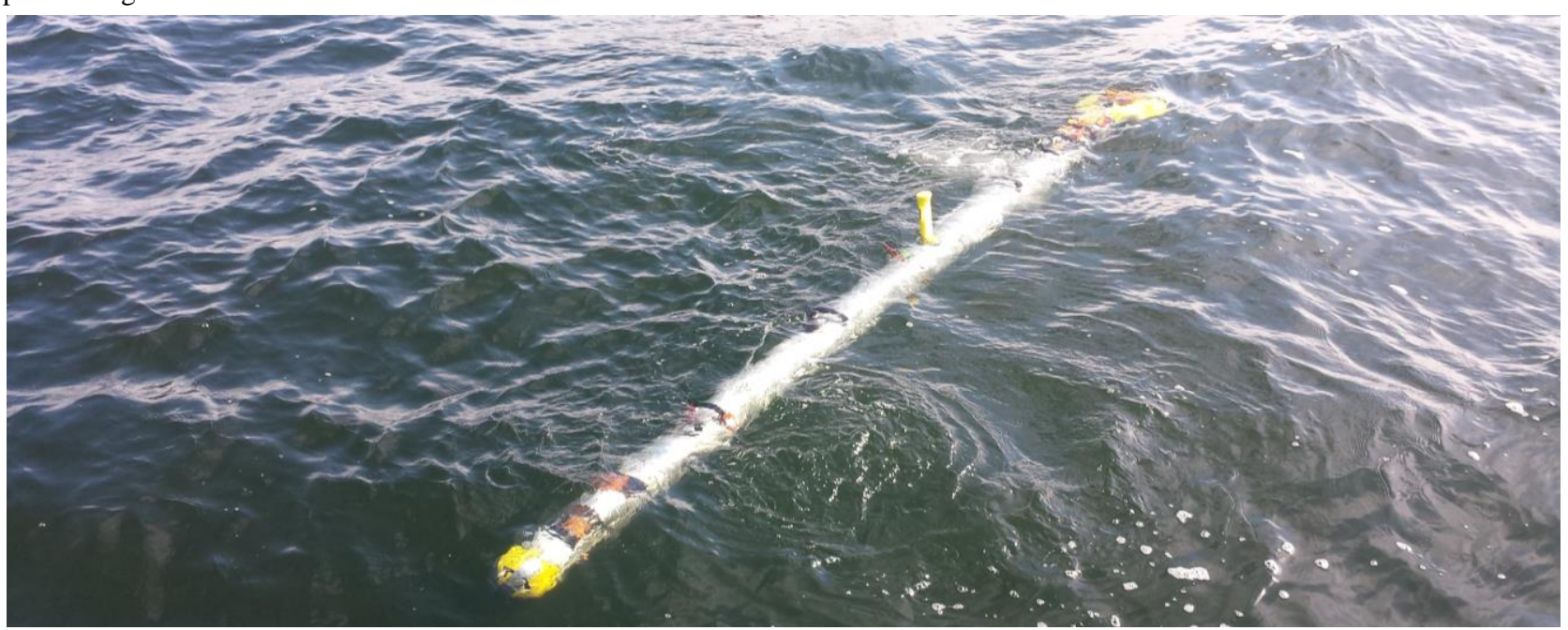

Figure 2. MARTA navigating in Rummu quarry

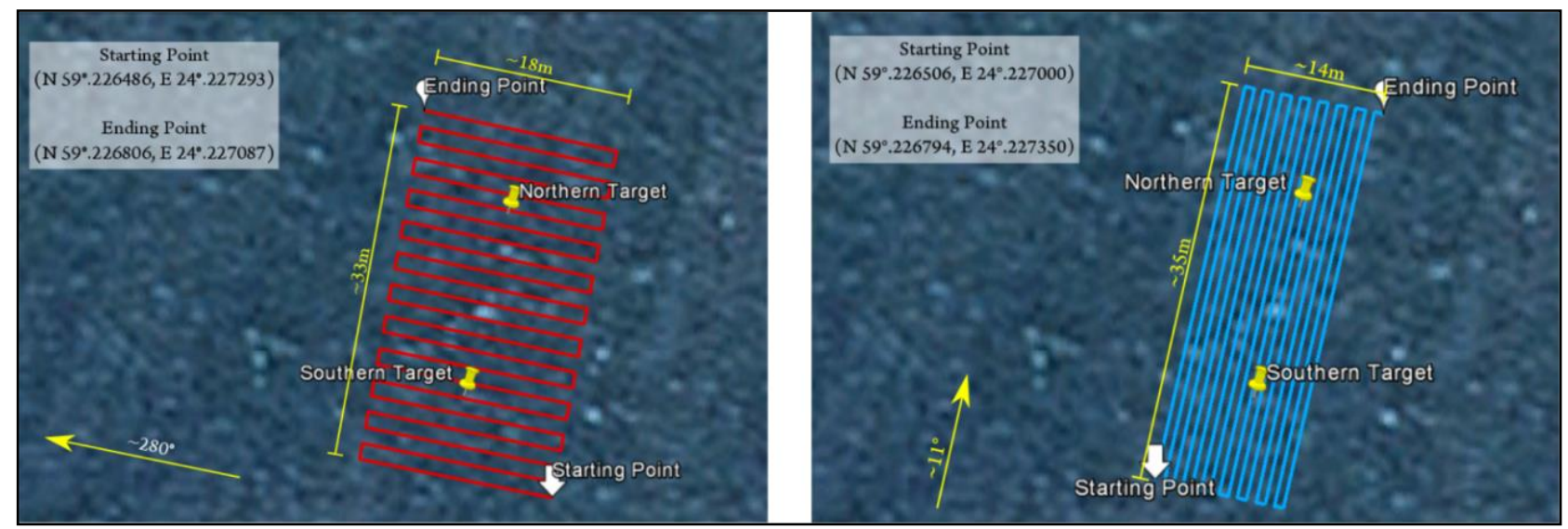

Figure 3. Layout of the two missions composing the Inspection campaign performed by MARTA AUV in Rummu quarry

they are not divers memories dependent.

The camera has captured a large amount of optical data installed on-board MARTA, and this resulted in the collection of important details of the lake bottom. Accurate 3D models of the objects have been obtained by processing optical data by advanced photogrammetry methods, such as Structure From Motion (Hartley et al, 2004), (Drap et al., 2008). The method allows estimating the $3 \mathrm{D}$ coordinates $\mathbf{x}_{\mathrm{ij}}$ of a world point using its known projections $\mathbf{x}_{\mathrm{ij}}$ on the various camera planes (here defined by the subscript $\mathbf{j}$ ) in the sequence of the optical data. The link between the real world coordinates $\mathbf{X}_{\mathrm{i}}$, and the camera projections $\mathbf{x}_{\mathrm{ij}}$ is expressed as follows: procedure implements the joint estimation of the coordinates $\mathrm{Xi}$ and the matrices Pj.

Most of the approaches to underwater 3D reconstruction, also known as structure from motion, use feature-based camera motion estimation and sparse point clouds for structure representation. Simple feature-based approaches are limited in underwater environments. The authors, following the idea already presented in (Rossi et al., 2015), (Newcombe, 2014), choose to improve the motion estimation between frames with a cost function optimization over all pixels in the reference image. A direct method that exploits every image pixel is significantly more robust. The approach developed in (Newcombe, 2014) achieves both camera tracking and structure estimation in quasi-real-time without using feature detection 
and matching, defining a per-pixel optimization framework instead. The overall idea of the ongoing research is, therefore, to exploit the concepts developed in (Newcombe, 2014) to underwater applications. The challenges involve mostly dealing with the significantly worse visibility compared to air, which is a problem, especially for camera tracking. Apart from image pre-processing to attenuate degradation effects caused by water, new approaches are studied to tackle this problem. In the framework of the particular application, the clear water of the Rummu quarry allowed simplifying the problem, as the assumption brightness constancy across corresponding pixels could be reasonably assumed valid. This is generally not true, but it can hold across a short time period and can be improved performing photometric normalization of each frame. Inertial navigation systems are usually present on the used mobile robots; therefore, one of the adopted methods is to integrate the motion estimation from the INS with the camera tracker, making the 3D system more robust in losing track. The Rummu quarry dataset has been exploited following a twofold approach to satisfy two different typical needs of an archaeological campaign. During the mission, the involved scientists needed a fast feedback visualization of the environment in order to formulate hypotheses on the nature of the detected targets and to, potentially, decide actions about the mission, e.g. replanning the mission path or abortion. In this case, the quality of the outcome is a minor requirement concerning the speed of the process. After the fieldwork time, the same data has been recomputed with more precise off-the-shelf processing software in order to document the site under analysis with the highest possible quality without any requirement on the necessary elaboration time. This different approach is aimed at the production of 3D models of the inspected area for more accurate analysis by the archaeologists and the fruition by the general audience.

\subsection{Quasi Real-Time 3D Models for Target Classification and Mission Re-planning}

A real-time evaluation of the completeness and accuracy degree of the data gathered during the fieldwork is, thus, a fundamental step in the adopted procedure showed below in Figure 4.

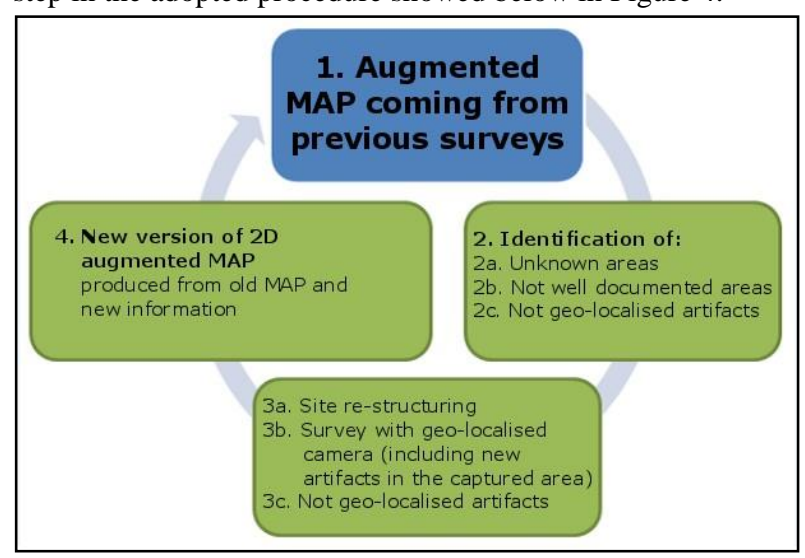

Figure 4. Typical work cycles for producing augmented map in archaeological campaign

This capability of the system is based on an Internet of Things (IoT) cloud structure, developed by the authors and called DiRAMa, devoted to acquired images collected from different internet connected devices and 3D reconstruction of the submarine environments. The major innovation introduced developing DiRAMa (Figure 5) is the integration of multiple heterogeneous information that came from the marine and underwater environment, using tested and reliable COTS components. The authors developed different hardware interfaces for most of the commercial DSLR, industrial, and action cameras in order to pre-filtering images and prepare them for the final computation (Scaradozzi et al., 2014). In the ARROWS project, a special block has been created to collect data from the MARTAs camera, calibrating the lens by means the producer optical information and a calibration panel for the case effect compensation as explain in (Drap et al., 2008) and obtain undistorted images set. Figure 5 represents a scheme of all DiRAMa components. In the scheme, single blocks are interconnected through lines that represent information exchange. Block A) Mobile acquisition device (LabVIEW software module in the case of MARTA) - This block characterizes each aspect related to the device used by the final user to collect data. Once on the surface, as soon as an Internet connection is available, the device becomes a sort of virtual buoy, sending data just collected to an opportune server. Block B) PHP/HTML5 Web server - A web server has been developed in order to acquire and catalog data.

Moreover, this block is responsible for communicating with 3D Engine Module in order to start reconstruction with user data and receive back results. Block C) 3D Engine Module and Database - The 3D Engine module is responsible for elaborations, implementing complex reconstruction algorithms, and talking to the block of the previous point. Block D) Cloud Manager - An added value of this project is constituted by the notification system, which allows the user to be informed of operations progress. Block E) Home Navigator - Users can visualize and modify their results using free software available on the network, such as MeshLab that can display models of three-dimensional geometries and the relative texture. Also the ability to provide reconstruction in pdf format the user can open with Adobe Acrobat Reader.

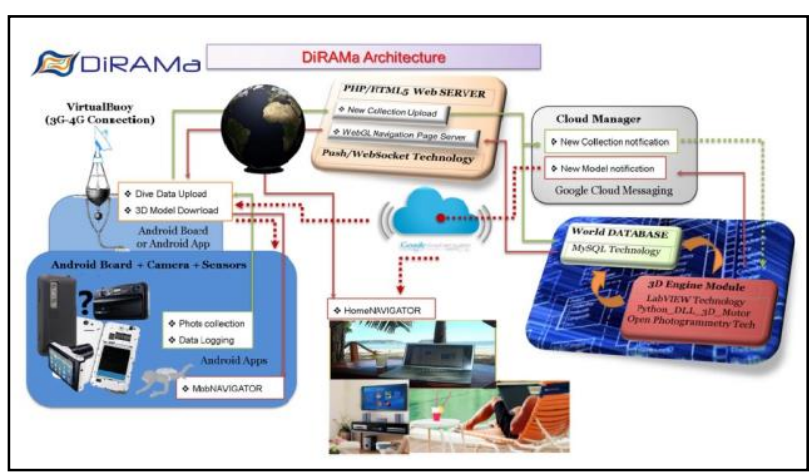

Figure 5. The DiRAMa Internet of Things (IoT) cloud structure

The 3D Engine has been asked to do in sequence the tree procedure (Point Clouds Identification, Build Geometry and Texturing) in order to obtain a draft reconstruction of the two targets under investigation within the end of the day work (July, 22nd). The computation characteristics and the results are presented in Table 1 and Table 2. Commercial software is used for image processing. Custom filters have been added to improve images before the $3 \mathrm{D}$ reconstruction. The computation power and time are critical issues. ROV and AUV are promising technologies if the algorithms could work on quasireal-time and, in this sense, Table 1 and 2 provide useful information for future improvements. 


\begin{tabular}{|l|l|l|}
\hline Target under investigation & Southern Target & Northern Target \\
\hline Number of photos & 119 & 107 \\
\hline Photos resolutions $(\mathrm{w} x \mathrm{~h})$ & $(2046 \mathrm{x} 1046)$ pixels x 96 dpi & $(2046 \times 1046)$ pixels x 96 dpi \\
\hline Number of key points & 11333 & 21063 \\
\hline RMS error & 1.18695 pixel & 1.20847 pixel \\
\hline Matching time & $7^{\prime} 17^{\prime \prime}$ & $21^{\prime \prime}$ \\
\hline Alignment time & $42^{\prime \prime}$ & $14^{\prime \prime}$ \\
\hline Densification result & 143445 key points & 310447 key points \\
\hline Faces count & 180000 & 180000 \\
\hline Vertices & 90270 & 90345 \\
\hline Texture format chosen & Square - 4096 pixels - color var uint8 & Square - 4096 pixels - color var uint8 \\
\hline Total processing time & $19^{\prime} 38^{\prime \prime}$ & $5^{\prime} 35^{\prime}$ \\
\hline
\end{tabular}

Table 1. The computation characteristics of the target under investigation. RMS error is computed, averaging the RMS of all the key points distance error. The distance taken into account is the one between the point on the image where the reconstructed 3D point is projected, and the original projection of that 3D point detected on the photo. The computer used for the computation is an Intel Core i7-2630QM-2GHz CPU with 32 GByte RAM, SSD HD, and Windows7-64bit OS



Figure 6. Semi Real-Time 3D reconstruction of the targets under investigations

\subsection{Complete High-Quality Model of the Target Area for Post Mission Fruition}

The same dataset collected during the Inspection Submission of July 22nd has been employed once more in an offline processing stage. Data has been processed again to produce more accurate, even though time-consuming, 3D representations of the same explored scenario. The goal was to generate a 3D model of the inspected area, including both the targets, with the highest possible quality, this time with more massive computation resources and no quasi-real-time constraint. The reconstructions have been generated exploiting Agisoft Photoscan version 1.0.4 build 1847 commercial software. The computation has been performed by means of an Intel CoreTM i7-4770 CPU @ 3.4 GHz, RAM 16 GB, Seagate Barracuda - 1 TB Serial-ATA 37200 rpm, Windows 10-64 bit OS. Benefiting from higher processing power, an enlarged set of pictures has been considered. This allowed to obtain an overall view of the inspected environment and to assess the relative spatial arrangement of the targets in addition to achieving a higher degree of detail of the model. Processing the dataset collected in the Rummu quarry using Structure from Motion resulted in the generation of a 3D point cloud (Figure 7) showing a portion of the lake bottom layer. The corresponding computation features are presented in Table 2.

\begin{tabular}{|l|l|}
\hline Target under investigation & Full Scene \\
\hline Number of photos & 2465 \\
\hline Photos resolutions (w x h) & 2046 x 1046 pixels \\
\hline Number of key points & 483590 \\
\hline RMS error & 0.78128 pixel \\
\hline Matching time & $22 \mathrm{~h} \mathrm{50}$, \\
\hline Alignment time & 2h 39' 25' \\
\hline Densification result & 15355371 key points \\
\hline Faces count & 2891599 \\
\hline Vertices & 1450334 \\
\hline Texture format chosen & 4096 x 4096, 24 bit depth \\
\hline Total processing time & 1d 5h 45' 58'” \\
\hline
\end{tabular}

Table 2. The computation features for the $3 \mathrm{D}$ reconstruction of the Rummu quarry machines. The computer used is an Intel CoreTM i7-4770 CPU @ 3.4 GHz, RAM 16 GB, Seagate Serial-ATA 37200 rpm, Windows 10-64 bit OS 
The result could be further refined by estimating the dense cloud and, in a later stage, the mesh surface fitting the point cloud. This has been performed exploiting popular open source software, i.e., Meshlab (Visual Computing Lab (CNR), 2008).

It is worth mentioning here that the primary step in the processing pipeline of the optical data concerns the correction of the distortions that are systematically introduced during the capture process due to the design of the camera and lens systems.

Following the approach adopted in the literature (Drap et al., 2007), the signal perturbation ascribed to the refraction effect is considered in the same way as the radial distortion caused by the light propagation through the lens system. Hence the authors decided to adopt a perspective camera model, extended to include radial distortion, whose parameters have been estimated following the Brown distortion model (Brown 1966), (Sedlazeck and Koch, 2012). The final result obtained by the Structure from Motion algorithm is further refined by the application of the bundle adjustment, the algorithm that simultaneously refines the scene geometry, camera poses, and parameters. Then, it has been possible to associate a metric to the obtained model, exploiting distance measurements between points easily identifiable on the acoustic images obtained through the SSS acquisitions performed during the Search Submission. The distance separating the two discovered machines has been estimated using the Sonar data captured by the inspecting AUV. This data has been geo-referenced using used in the past for the extraction of Vasalemma marble, is described. The underwater robots involved in this demonstration were a commercial AUV housing an interferometric SSS, and MARTA, an AUV built within the ARROWS project, with hovering capability and equipped with an optical camera. The novel aspects described in this paper and developed within the ARROWS project have been validated during the final demonstration of the project. On the one hand the Search and Inspection strategy applied to the field of underwater archaeology demonstrated to be a suitable way to individuate potential artifacts, to classify them as interesting targets or not, and to acquire images for the reconstruction of 3D models. Other studies on different sites convince authors of the method potential (Allotta et al., 2018), (Scaradozzi et al., 2014), and (Zingaretti et al., 2018) and the solutions presented here demonstrate replicability.

On the other hand, the experimentation described in the paper validated the use of MARTA AUV, developed within the ARROWS project, as a highly modular vehicle (both from a payload and a propulsion system point of view) according to the guidelines by expert marine archaeologists. MARTA can adapt its characteristics to the different exigencies of an archaeological campaign through the addition/removal of different modules. The configuration adopted in the described experiment was suitable for the role of Inspection vehicle, thus including a set of propellers for hovering capabilities and highresolution cameras as payload. The ARROWS AUVs, with

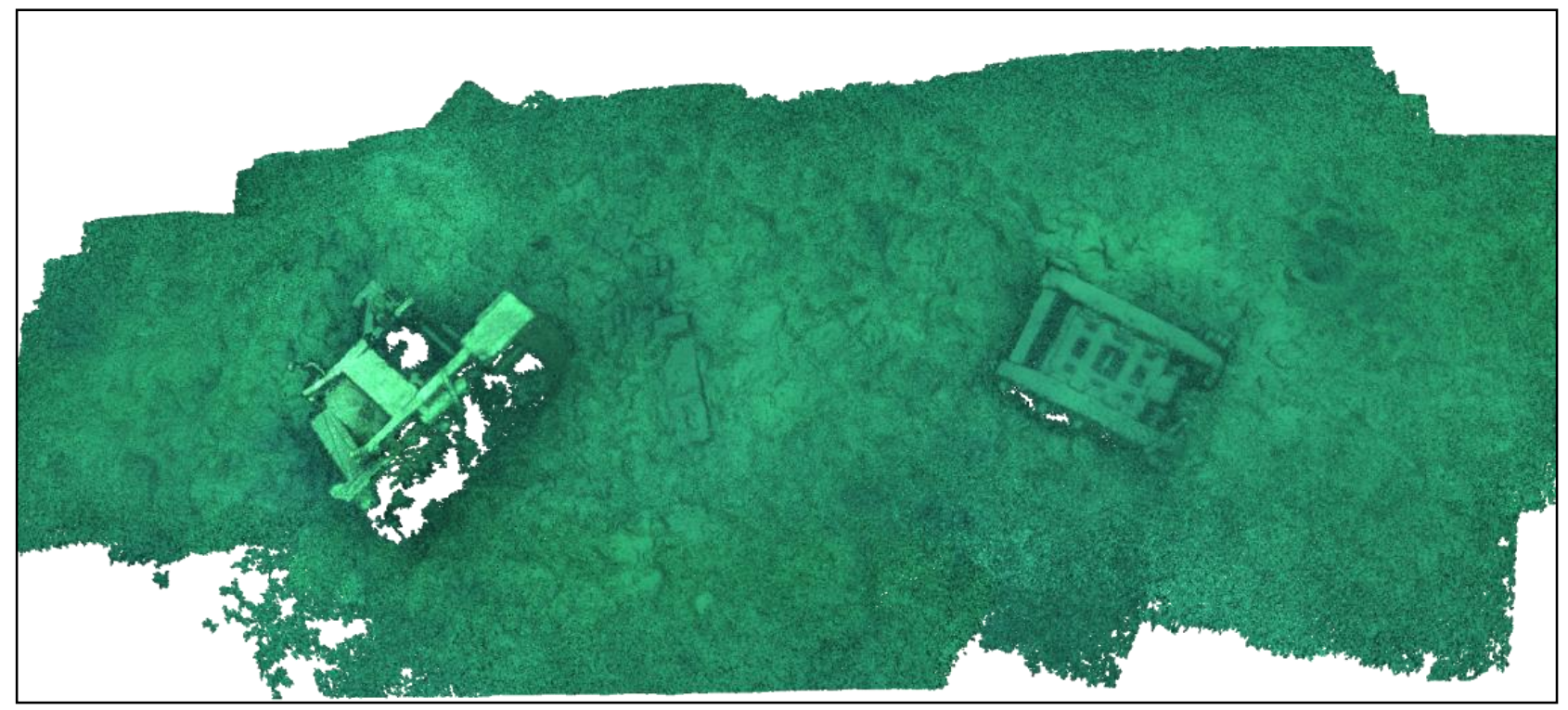

Figure 7. Point cloud generated from the offline processing of the Rummu dataset

the positioning sensors aboard the vehicle, hence providing exact ground reference. The 3D models obtained during the project are available on the ARROWS project website (http://www.arrowsproject.eu) in the Media Center section ${ }^{3}$.

\section{CONCLUSIONS AND FURTHER DEVELOPMENTS}

The research paper focused on the final demonstration of the European ARROWS project in Estonia: the Search and Inspection mission in Rummu quarry, a submerged mining site different specific roles, were able to exploit their payload sensors to localize two targets, particularly interesting for their important dimension, and to acquire detailed images of them. The collected images were used for two different elaboration processes. First use was the generation of a 3D model of the two targets, allowing evaluations on the targets nature and the potential necessity of further acquisitions. This first process, based on the DiRAMa IoT cloud structure, required a limited amount of time to give an outcome to the archaeologists during the mission day. It thus represents an essential tool for the archaeologists: they can plan the activities of the following day of the campaign, based on feedback from the just-completed mission. The second process was oriented to a high-quality $3 \mathrm{D}$ model of the inspected area without strict time constraints. This phase is based on the use of commercial software with the final goal of including the new 3D model of the inspected scene in a 
virtual navigation environment: this can simplify and improve the dissemination and the fruition of the underwater cultural heritage.

Further improvements to the system for 3D model reconstruction concern the exploitation of the estimates of the vehicle position, velocity, altitude and orientation angles provided by the navigation sensors installed onboard the vehicle, e.g., DVL, IMU, and gyrocompass sensors, which are correctly fused using the above-mentioned methods. The pose measurements provided by such sensors can be exploited to transform the coordinate points of the reconstructed points from the local camera frame to a geo-referenced world frame. This will enrich the 3D reconstruction process results by incorporating more accurate metric properties in the system than the one retrieved from the acoustic images acquired using the SSS. This will enable direct accurate metric measurements within the recreated virtual environment.

\section{ACKNOWLEDGEMENTS}

This work has been supported by the European ARROWS project, that has received funding from the European Union's Seventh Framework Programme for research, technological development and demonstration under grant agreement no. 308724 .

\section{REFERENCES}

Allotta, B., Costanzi, R., Ridolfi, A., Salvetti, O., Reggiannini, Kruusmaa, M., Salumae, T., Lane, D. M., Frost, G., Tsiogkas, N., Cocco, M., Gualdesi, L., Lacava, G., Roig, D., Gundogdu, H T., Dede, M I C., Baines, S., Tusa, S., Latti, P., Scaradozzi, D., 2018. The ARROWS Project: robotic technologies for underwater archaeology. IOP Conf. Ser.: Mater. Sci. Eng.

Allotta, B., Baines, S., Bartolini, F., Bellavia, F., Colombo, C., Conti, R., Costanzi, R., Dede, C., Fanfani, M., Gelli, J., Tolasa Gundogdu, H., Monni, N., Moroni, D., Natalini, M., Pascali, M. A., Pazzaglia, F., Pugi, L., Ridolfi, A., Reggiannini, M., Roig, D., Salvetti, O., Tekdemir, E. I., $2015 \mathrm{~b}$. Design of a modular Autonomous Underwater Vehicle for archaeological investigations. Proceedings of MTS/IEEE OCEANS 2015, Genova, Italy, May 2015.

Allotta, B., Costanzi, R., Pascali, M. A., Reggiannini, M., Ridolfi, A., Salvetti, O., Sharvit, J., 2015c. Acoustic data analysis for underwater archaeological sites detection and mapping by means of autonomous underwater vehicles. Proceedings of MTS/IEEE OCEANS 2015, Genova, Italy, May 2015.

Allotta, B., Costanzi, R., Magrini, M., Monni, N., Moroni, D., Pascali, M. A., Reggiannini, M., Ridolfi, A., Tampucci, M., Salvetti, O., $2015 \mathrm{~d}$. Towards a robust system helping underwater archaeologists through the acquisition of georeferenced optical and acoustic data. Proceedings of Springer 10th International Conference on Computer Vision Systems, ICVS 2015, Copenhagen, Denmark, July 2015.

AUVAC Database, 2016: Retrieved January 19, 2016, from http://www. auvac.org/.

Brown, D. C., 1966 . Decentering Distortion of Lenses. Photogrammetric Engineering, 32(3), 444-462.
Conte, G., Gambella, L., Scaradozzi, D., Zanoli, S. M., Caiti, A., Calabrò, V., Alcocer, A., Alves, J., Cardeira, B., Cunha, R., Curado, F., Oliveira, P., Oliveira, A., Pascoal, A., Rufino, M., Sebastio, L., Silvestre, C., 2009. Underwater vehicle technology in the European research project VENUS. Underwater Technology, 28, 175-185.

Conte, G., De Capua, G. P., \& Scaradozzi, D., 2012. Modeling and control of a low-cost ASV. IFAC Proceedings Volumes, 45(27), 429-434.

Drap, P., Seinturier, J., Scaradozzi, D., Gambogi, P., Long, L., Gauch, F., 2007 . Photogrammetry for Virtual Exploration of Underwater Archaeological Sites. Proceedings of the 21st CIPA symposium, AntiCIPAting the future of the cultural past, Athens, Greece.

Drap, P., Scaradozzi, D., Gambogi, P., \& Gauch, F., 2008. Underwater Photogrammetry for Archaeology-The Venus Project Framework. In GRAPP, 485-491.

Freitag, L., Grund, M., Von Alt, C., Stokey, R., Austin, T., 2005 . A shallow water acoustic network for mine countermeasures operations with autonomous underwater vehicles. Underwater Defense Technology(UDT).

Hartley, R. I., Zisserman, A., 2004. Multiple View Geometry in Computer Vision. Second Edition, Cambridge University Press.

Lowe, D. G., 2004. Distinctive Image Features from Scale Invariant Keypoints. Journal International Journal of Computer Vision, 60(2), 91-110, Kluwer Academic Publishers Hingham, MA, USA, November 2004.

Ludvigsen, M., Sortland, B., Johnsen, G., Singh, H., 2007. Applications of Geo-Referenced Underwater Photo Mosaics in Marine Biology and Archaeology. Oceanography, 20, 140-149.

Martin, C. J. M., Martin, E. A., 2002. An underwater photomosaic technique using Adobe Photoshop. International Journal of Nautical Archaeology, 31(1), 137147.

Morganti, G., Perdon, A. M., Conte, G., \& Scaradozzi, D., 2009. Multi-agent system theory for modelling a home automation system. In International Work-Conference on Artificial Neural Networks, Springer, Berlin, Heidelberg.

Mugnai, F., Lombardi, L., Tucci, G., Nocentini, M., Gigli, G., and Fanti, R., 2019. Geomatics in bridge structural health monitoring, integrating terrestrial laser scanning techniques and geotechnical inspections on a high value cultural heritage. Int. Arch. Photogramm. Remote Sens. Spatial Inf. Sci., XLII2/W11, 895-900.

Newcombe, R. A., 2016. Dense Visual SLAM. Ph.D. dissertation, Imperial College London, 2014.

OceanServer IVER3, 2016. http://www.iverauv.com/Iver3_AUV_Brochure.pdf. 
Pizarro, O., Singh, H., 2003. Toward Large-Area Mosaicing for Underwater Scientific Applications. IEEE Journal of Oceanic Engineering, 28(4), 651-672.

Rossi, M., Scaradozzi, D., Drap, P., Recanatini, P., Dooly, G., Omerdic, E., Toal, D., 2015. Real-time reconstruction of underwater environments: from 2D to 3D. Proceedings of MTS/IEEE Washington OCEANS 2015.

Scaradozzi, D., Sorbi, L., Zoppini, F., 2014. DiRAMa facilitates data gathering and analysis at sea system creates 3D images using data gathered from mobile devices. Sea Technology Journal, 55(6), 19-22.

Sedlazeck, A., Koch, R., 2012. Perspective and Nonperspective Camera Models in Underwater Imaging Overview and Error Analysis. Outdoor and Large-Scale Real-World Scene Analysis, Dagstuhl Castle, 212-242.

Sorbi, L., Scaradozzi, D., Zoppini, F., Zingaretti, S., Gambogi, P., 2015. Robotic tools and techniques for improving research in an underwater delicate environment. Marine Technology Society Journal, 49(5), 6-17.

Van Damme, T., 2015. Computer Vision Photogrammetry for Underwater Archaeological Site Recording in a LowVisibility Environment. The International Archives of the Photogrammetry, Remote Sensing and Spatial Information Sciences, XL-5/W5, 231-238.

Visual Computing Lab, 2018 . Meshlab: an open source 3D mesh processing system. Visual Computing Lab. http://meshlab.sourceforge.net/.

Zingaretti, S., Scaradozzi, D., Ciuccoli, N., Costa, D., Palmieri, G., Bruno, F., ... \& Manglis, A., 2018. A Complete IoT Infrastructure to Ensure Responsible, Effective and Efficient Execution of Field Survey, Documentation and Preservation of Archaeological Sites. In 2018 IEEE 4th International Forum on Research and Technology for Society and Industry (RTSI). 